\title{
1 Sexualität in Pubertät und Jugendalter
}

\author{
Burkhard Brosig
}

Im folgenden Kapitel sollen zentrale Themen der Jugendsexualität behandelt werden: Neben der Psychodynamik der jugendlichen Sexualentwicklung als einer psychologischen Metatheorie sollen die Bereiche Aufklärung, auch hinsichtlich der Prophylaxe von HIV-Infektionen, Masturbation, „Das erste Mal“ und schließlich, eher skizzenhaft, Themen zu Partnerschaftsverhalten und Homosexualität erörtert werden (vgl. Euler u. Brosig 2004).

\subsection{Pubertät und Adoleszenz}

Pubertät im medizinischen Sinne wird meist verstanden als die Zeitspanne der Geschlechtsreifung, beginnend in der späten Kindheit, unter anderem mit der Ausbildung der sekundären Geschlechtsmerkmale, und endend mit der ersten Regelblutung (Menarche) beim Mädchen und der ersten Ejakulation (Ejakularche) beim Jungen. Die Pubertät bezieht sich also im weitesten Sinne auf die physiologische Entwicklung vom nicht-reproduktionsfähigen Kind zum reproduktionsfähigen Erwachsenen. Der Eintritt in die Geschlechtsreife bedeutet psychisch gewissermaßen den Beginn einer „neuen Zeit“: Er bringt die Gewissheit, aber auch die mit Konflikten behaftete Erkenntnis mit sich, eindeutig dem männlichen oder weiblichen Geschlecht anzugehören. Dabei stehen nach Freud (1905) somatische und psychische Entwicklungsvorgänge zunächst unverknüpft nebeneinender.

Das Jugendalter wird im psychosexuellen Kontext als Adoleszenz bezeichnet. Diese Epoche markiert den sich an die Geschlechtsreife anschließenden Entwicklungsprozess. Er umfasst stärker die psycho-sozio-kulturelle Komponente des Jugendalters, also einer Entwicklung, die sich an somatischen Gesichtspunkten orientiert.

Der für Pubertät und Adoleszenz angegebene Altersbereich variiert vom Beginn im 9. Lebensjahr bis zum Ende um das 25. Lebensjahr. Orientierung gebende „Fixpunkte" sind nach Kluge (1998) Menarche (Median 12,2 Jahre) und Ejakularche (Median 12,6 Jahre). Die Darstellung im folgenden Text soll sich überwiegend auf den Zeit- 
raum zwischen dem 14. und dem 17. Lebensjahr beziehen. Mädchen und Jungen in diesem Altersbereich stellen normalerweise auch die Stichprobe bei Repräsentativerhebungen zum Sexualverhalten Jugendlicher dar (z.B. Bundeszentrale für gesundheitliche Aufklärung, Kluge 1998). Jugendliche können bei der initialen Aufnahme sexueller Aktivität nicht auf eigene praktische Erfahrungen zurückgreifen. Im Gegensatz zu anderen „Lernbereichen“ steht ihnen dabei zudem in der Regel kein „Lehrender" zur Verfügung. Dennoch sind Jugendliche mit normativen Rahmenbedingungen konfrontiert (Medien, Schule, Eltern, Freunde). Nach Trautner (1996, S. 165) ist der Jugendliche mit folgenden „Entwicklungsaufgaben“ (vgl. Ewert 1983, S. 55) konfrontiert:

- Akzeptanz des eigenen weiblichen bzw. männlichen Körpers

- Aufbau der sexuellen Orientierung in der Partnerwahl („sex“)

- Rollen-Neudefinition in der peergroup in Bezug auf beide Geschlechter

- Auseinandersetzung mit der gesellschaftlich determinierten Geschlechtsrolle (,gender“) in Beruf und Familie

Aus der Perspektive des Schriftstellers beschreibt Hubert Fichte (1974) in seinem „Versuch über die Pubertät“ die Entwicklungskrisen des Jungen.

\subsection{Zur Psychodynamik der jugendlichen Sexualentwicklung}

Nach dem psychoanalytischen Modell folgt auf die Latenzzeit (6.-12. Lebensjahr), welche die infantilen Sexualität in der Auflösung des Ödipuskomplexes ablöst, die pubertäre und schließlich adoleszentäre Entwicklung. Nach Freud (1905) ist die Pubertät zu verstehen als die endgültige Überführung des infantilen Sexuallebens in seine adulte Gestaltung mit drei Zielen: Unterordnung der erogenen Zonen unter das Primat der Genitalität, neue, für beide Geschlechter verschiedene, Sexualziele sowie schließlich die differente Wahl von Sexualobjekten außerhalb der Familie. In der Vorpubertät brechen bereits kontrolliert geglaubte Triebneigungen wieder durch. Wie in der frühen Kindheit stehen sich durch diesen Triebdurchbruch von Neuem ein schwaches Ich als neurophysiologische Kommandozentrale des Menschen, und ein starkes Es als Hort der biologischen Motive und Triebe gegenüber. Sigmund Freud definiert infantile und pubertäre Sexualität als zweizeitigen Ansatz der Sexualentwicklung beim Menschen (Freud 1905, S. 135).

Die seelische Dynamik in der Pubertät ist aus der Sicht der Psychoanalyse zum einen Wiederholung kindlicher Sexualentwicklung, zum anderen auch deren Fortführung auf einem neuen Niveau (vgl. hierzu Ewert 1983; Freud 1958; Lerner 1987). Was man von außen als Devianz im Sozialverhalten interpretieren mag, stellt lediglich den Ausdruck dieses Kampfes dar. Der Jugendliche

„wehrt seine Triebregungen ab, gibt ihnen aber auch nach; er vollbringt Wunder an Selbstbeherrschung, ist aber auch Spielball seiner Gefühle, er liebt seine Eltern und hasst sie zugleich, er ist gleichzeitig voller Revolte und voller Abhängigkeit; er will nichts von seiner Mutter wissen, sucht sie aber unvermittelt zu vertraulichen Aussprachen; er ist bereit, sich selbst aufzugeben und anderen hörig zu werden, sucht aber auch gleichzeitig seine eigene Identität; er hat mehr künstlerisches Verständnis, ist idealistischer, großzügiger und uneigennütziger als je vorher oder nachher; aber er ist auch das Gegenteil: egoistisch, selbstsüchtig und berechnend" (Freud 1958, S. 1768). 
Die Disharmonie in der psychischen Struktur während der Pubertät mit den schweren Konflikten, die sich zwischen Ich und Es abspielen, wird gewissermaßen als Heilungsvorgang gesehen, einhergehend mit der Notwendigkeit, die alte Struktur zu zerstören.

Weiterhin erfolgt im Rahmen der Pubertät der Übergang von der Selbstliebe zur Liebe anderer: Die narzisstische Ich-Libido wird zur Objektlibido. Während der Latenzzeit hat sich eine „Inzestschranke“ errichtet, die es dem Jugendlichen verbietet, die geliebten Objekte seiner Kindheit zu Sexualobjekten zu wählen (Freud 1905, S. 126). Diese Inzestschranke ist dann auch verantwortlich dafür, dass die in der Pubertät reetablierte Sexualität des Kindes von den Eltern weg auf andere, wenngleich oft auch ähnliche Objekte gelenkt wird. Die Verwerfung dieser inzestuösen Phantasien stellt dabei gleichzeitig eine der bedeutsamsten, jedoch auch schmerzhaftesten psychischen Leistungen der Pubertät dar. Losgelöst von präödipalen und ödipalen Beziehungsmustern sucht der Jugendliche die Identifikation mit außerfamiliären Figuren, Jugendgruppenleitern, Lehrern, Stars und gelangt so zur sozialen Rollenfindung im Sinne einer Identifikation mit dem gleichgeschlechtlichen Elternteil. Die „peergroup“ der Gleichaltrigen stellt dabei gewissermaßen ein Übergangsobjekt zum Ersatz der elterlichen Fürsorge dar.

Fehlt die Integration triebhaft-wilder Aspekte der Selbstfindung mit sozial konformen Rollenmustern und überwiegt eine angepasste äußere Schale unter Abspaltung vitaler Elemente der Persönlichkeit, so spricht man vom Konzept des „falschen Selbst“ (Winnicott 1965). Hier dominiert eine Fassade der Aspontaneität und Unechtheit.

\subsection{Aufklärung}

Auf die Veränderungen des kindlichen Körpers und die damit einhergehenden Stürme im seelischen Erleben des Pubertierenden sollte, idealiter, eine adäquate Aufklärung vorbereiten. Nach den Daten der Bundeszentrale für Gesundheitliche Aufklärung (BzgA 2001) waren im Jahre 2001 immerhin 65\% der untersuchten Jungen über die sexuellen Crundtatsachen aufgeklärt, 1980 gaben dies noch weniger als die Hälfte an. Ähnliche Aufklärungsquoten wurden für die Mädchen bestimmt.

Obwohl in den Vereinigten Staaten von Amerika sowohl Jugendliche als auch Eltern fast ausnahmslos davon überzeugt sind, dass die Eltern die Rolle der „Aufklärer“ übernehmen sollen, tun es diese dort jedoch in den seltensten Fällen (Gordon u. Gilgun 1987). Meist wird, offenbar im Gegensatz zu den Verhältnissen in Deutschland, diese Rolle von „peers“ übernommen, was häufig nicht nur zu Fehlinformationen, sondern auch zur Verknüpfung mit entsprechenden pro-sexuellen Appellen und sozialem Druck bezüglich einer frühen Aufnahme von sexuellem Verhalten führt. Auch sei in diesem Kontext ausreichende Information bezüglich der Kontrazeption nicht zu erwarten. Folgende Themen erscheinen zentral wichtig:

\subsubsection{Masturbation}

Kinsey et al. veröffentlichten bereits 1948 Zahlen über die „Prävalenz“ der Onanie, dort definiert als ,,jede Art der vorsätzlichen Selbststimulierung, die dazu bestimmt ist, erotische Erregung hervorzurufen“ (S. 450). 92 Prozent der Männer betreiben demnach Onanie bis zum Orgasmus, 82 Prozent bereits vor dem 15. Lebensjahr, mit 
erheblichen interindividuellen Unterschieden bezüglich der Frequenz und mit einer kontinuierlichen Abnahme von der Pubertät bis ins höhere Lebensalter. Zu ähnlichen Zahlen kommen auch später durchgeführte deutsche Studien (vgl. hierzu Clement 1986; Schnack u. Neutzling 1996).

Ein besonderer Mythos stellt die „Pollution“ dar: Teilweise wird für den Eintritt in die Geschlechtsreife bei Jungen nicht die Ejakularche als Maß genommen, sondern die Pollution. Pollution (von lat. polluere: beflecken) ist der erste (unwillkürlich erfolgende), nächtliche Samenerguss. Zieht man hierzu repräsentative Daten heran, so zeigt sich, dass überdurchschnittlich viele Jungen die erste Ejakulation jedoch gar nicht in diesem Rahmen erleben. Zwei Drittel der Jungen gelangen durch Onanie zur ersten Ejakulation und nicht etwa durch Pollution (Kinsey et al. 1948). Nach Kluge (1998) hatten immerhin nur 43 Prozent der Jungen „zufällig“ nachts ihren ersten Samenerguss. Bei Schnack u. Neutzling (1993, S. 193) heißt es dazu:

„Wer den ersten Samenerguss erlebt hat, der ist sozusagen in den Club der reifen Sexualwesen aufgenommen, der ist nicht mehr Kind. (...) Deshalb warten die meisten Jungen auch nicht ab, bis ihnen die Eintrittskarte in der Nacht geschenkt wird“.

Auch Kinsey et al. (1948) stellten bereits fest, dass „die Onanie in späterem Alter keinen Schaden anrichtet“ (S. 455). Das „gilt auch für die aktivsten Männer“ (S. 457). Für ihn war Onanie schon damals als etwas zu sehen, was „nervöse Spannungen“ beseitigt. Jugendliche, die onanieren, „führen im Ganzen gesehen ein ausgewogeneres Leben“" (S. 467).

Die von Kinsey dargelegten gesellschaftlich etablierten Vorstellungen über die Masturbation sind heute längst überholt. Umso mutiger erscheint die literarische Verarbeitung der Thematik Ende des 19. Jahrhunderts durch Arthur Rimbaud in den „Läusesucherinnen“ (Rimbaud 1907). Trotz befreiter und befreiender Lust sind Jungen, die ihre Sexualität neu entdecken, auch heute noch gewissermaßen „eingeklemmt “ in der Zweischneidigkeit der damit verbundenen Erlebniswelt: Auf der einen Seite steht der praktisch unkontrollierbare Drang zur Onanie, den viele pubertierende Jungen wie eine Sucht erleben, auf der anderen Seite stehen nicht selten Schuldgefühle und das Verlangen, sich besser im Griff zu haben: „Gefühle der Leere, Ernüchterung, Einsamkeit und nicht selten auch Wut und Hass gegen sich selbst kennen fast alle Jungen, nachdem sie onaniert haben“ (Schnack u. Neutzling 1993, S. 194). Wenn auch heute die meisten Jungen nicht mehr daran glauben, dass sie durch die Masturbation „verblöden“, so ist doch eine Sorge unter den „Weltmeistern“ (Schnack u. Neutzling 1996, S. 136) im Masturbieren immer noch weit verbreitet: Viele Jungen glauben, nur zu einer limitierten Anzahl von Ejakulationen fähig zu sein.

Die Ejakularche bei den Jungen ist, analog zur Menarche bei den Mädchen, ein zentraler - wenn nicht sogar der zentrale - Punkt in der jugendlichen sexuellen Entwicklung. Dabei wird der erste Samenerguss von Jungen wesentlich positiver erlebt als die erste Regelblutung von Mädchen. Jungen können den Eintritt der Geschlechtsreife durch Selbstbefriedigung selbst „herbeiführen“ und erleben ihn so häufiger als „schön“, während Mädchen von der ersten Regelblutung eher überrascht werden und diese häufig als unangenehm oder zwiespältig erleben: „Die sexuelle Initiation hat für die Mädchen mit Blut zu tun, für die Jungen mit Lust“ (Schnack u. Neutzling 1996, S. 153). Bei den Jungen ist dann auch der Anteil derer, die den ersten Samenerguss als angenehm empfinden, bei denjenigen signifikant höher, die Masturbationserfahrung haben, als bei denjenigen ohne solche Erfahrungen (vgl. hierzu Kluge 1998). 


\subsubsection{Das „erste Mal“}

Das Durchschnittsalter für den ersten Geschlechtsverkehr (Kohabitarche) beträgt für Mädchen 14,9, für Jungen 15,1 Jahre (aus der Gruppe der 14-17-Jährigen, ohne die zu diesem Zeitpunkt noch immer Koitus-Unerfahrenen, zitiert nach den Daten der BZgA 2001), bei Plies et al. (1999) finden sich Zahlen von 16,7 \pm 1,7 Jahren (hier 14-24-Jährige, ebenfalls ohne Koitus-Unerfahrene) für beide Geschlechter. Im Vergleich zu früheren Erhebungen bestehen keine wesentlichen Unterschiede mehr zwischen Mädchen und Jungen. Jeder dritte Jugendliche zwischen 14 und 17 hat somit schon Geschlechtsverkehr gehabt. Etwas niedrigere Werte fand Kluge (1998): Von den 14-17-jährigen Jungen in seiner Stichprobe hatten nur 26\% ihren ersten Geschlechtsverkehr bereits erlebt, die meisten mit 15 oder 16 Jahren.

Die Zunahme früherer Koitus-Erfahrung im Vergleich zu früheren Erhebungen (vgl. dazu auch Clement 1986) bezieht sich somit vorzugsweise auf die Jungen, was die Angleichung an die Quote der Mädchen erklärt. Unter den 14-Jährigen hatte jeder Zehnte, unter den 17-Jährigen zwei Drittel bereits ihren ersten Geschlechtsverkehr (BZgA 2001). Dass allerdings nicht nur die sexuelle Aktivität der Jungen relativ zu den Mädchen früher erfolgt, sondern Jugendliche auch insgesamt früher koitale Erfahrungen machen, zeigt der Vergleich zu älteren Befragungen (s. Abb. 1).

Plies et al. (1999) geben die Anzahl der Geschlechtspartner bei den Jungen und Mädchen ihrer Stichprobe (14-24-Jährige) mit zum Zeitpunkt der Befragung als „meist 1-2“ an. Der erste GV ist dabei immer häufiger ungeplant: $34 \%$ der Jungen und 25\% der Mädchen sind davon „völlig überrascht“ worden (BZgA 20o1). Die Autoren sehen in diesem Verhalten den Hauptgrund für die konstant hohe Quote der „Nichtverhütung“ beim „ersten Mal“.

Ewert (1983) bezeichnet die „Vorverlagerung“ der sexuellen Aktivität über das 20. Jahrhundert hinweg als „säkulare Akzelaration“ (S. 87) mit einem Beginn in den 2oerJahren und seinem Höhepunkt in den Goern. Die Entkrampfung und Liberalisierung der geschlechtlichen Aktivität im Jugendalter fand insbesondere in den Goer-Jahren

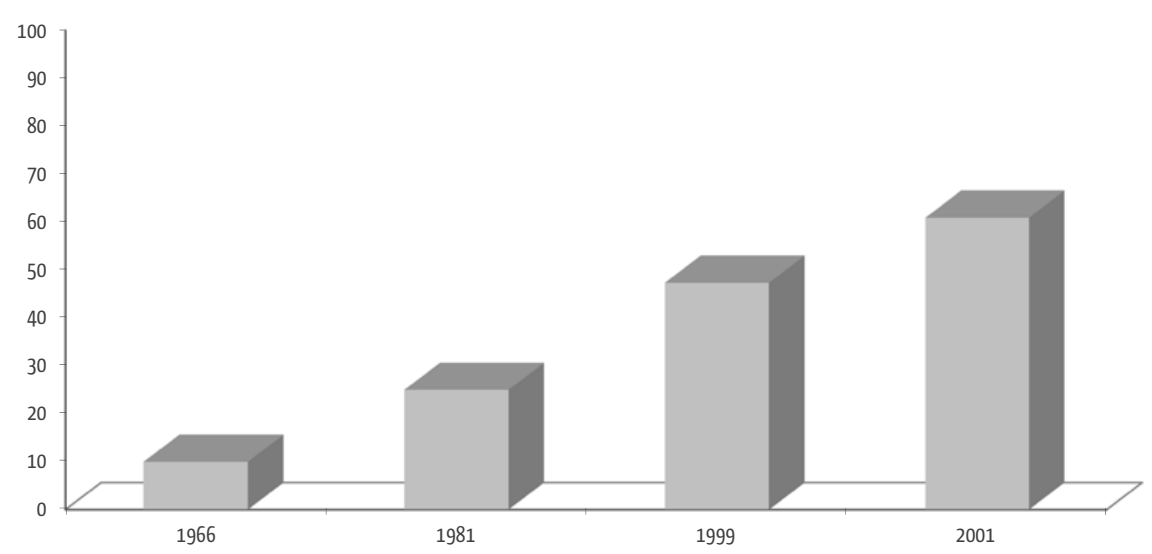

Abb. 1 Stattgehabte Kohabitarche von Jungen im 17. Lebensjahr in Deutschland (in Prozent): Zahlen für 1966 und 1981 nach Clement (1986), für 1999 nach Plies et al. (1999), für 2001 nach BzgA (2001) 
im Kontext der als „freudomarxistisch“ zu charakterisierenden Bewegung der sexuellen Befreiung statt. Dies führte in der Folge jedoch nicht automatisch zu größerer Promiskuität, sondern eher zu einer Internalisierung von Werten wie Rücksichtnahme auf den Partner und die bestehende Liebesbeziehung im Kontext einer neuen, selbstgewählten Treue geführt. Damit im Einklang sieht Plies et al. (1999) auch die Partnerideale der jungen Generation mit offenbar keineswegs altmodischen Begriffen wie „Liebe“, „Vertrauen“ und „Treue“.

Nach den Daten der BzgA erleben 80\% der Jungen, jedoch nur 58\% der Mädchen den ersten GV als „etwas Schönes“. Ähnliche Zahlen fand auch Kluge (1998), nach dem mehr als drei Viertel der Jungen im Vergleich zu nur drei Fünftel der Mädchen das „erste Mal“ als schön empfanden. Der aus früheren Studien bereits bekannte Geschlechtsunterschied im Erleben des ersten Koitus besteht demnach weiterhin.

Amerikanische Autoren (Gordon u. Gilgun 1987) ermittelten empirisch, dass 13-19-jährige Mädchen nach dem „ersten Mal“ eher Schuld, Angst und Verletzung empfanden, wohingegen Jungen sich als glücklich und zufrieden bezeichneten. Insgesamt geben zweimal so viele Jungen wie Mädchen positive Gefühle an. Bei Mädchen ist wiederum die Liebe zum Partner sehr viel häufiger als bei Jungen das Motiv für die Aufnahme der ersten sexuellen Aktivität.

\subsection{Kontrazeption und HIV-Prävention}

Jugendliche sind eine besondere Zielgruppe von Anti-Aids-Kanpagnen, und zwar allein schon deshalb, weil sie nicht einem Prozess des „Umdenkens“ unterliegen; es geht vielmehr um das neue Erlernen von Verhaltensweisen. Dabei bleiben die ersten koitalen Erfahrungen der „wunde Punkt“ der Verhütung, da die Anwendung des Kondoms nach wie vor eine zusätzliche Hürde in dieser Intimsituation darstellt: Wer beim ersten Mal nicht verhütet, hat auch später eine größere Wahrscheinlichkeit für ungeschütztes Sexualverhalten. Dass die Anti-Aids-Kampagnen dennoch bei Jugendlichen erfolgreich waren, zeigen folgende Zahlen: Die Nichtanwendung von Verhütungsmitteln beim „ersten Mal“ hat sich seit 1980 bei Jungen etwa halbiert (1980: 29\%, 2001: 15\%), wobei seit den goer-Jahren keine weitere Verringerung der Quote der nichtverhütenden Jungen erreicht werden konnte. Prädiktoren für die Nicht-Verhütung beim ersten Geschlechtsverkehr sind Bildung (höhere Quote bei unterem und mittlerem Bildungsniveau), Alter (je jünger desto nachlässiger), der Grad der Vertrautheit mit dem Sexualpartner (Nicht-Verhütung besonders bei flüchtiger Bekanntschaft) und der Umgang mit Sexualität und Verhütung im Elternhaus: je offener der Umgang, desto ausgeprägter die Verhütungsbereitschaft (BZgA 2001; Plies et al. 1999).

Das Kondom fand als Methode der Verhütung beim „ersten Mal“" 1980 nur bei 28\% der Jungen Anwendung, 2001 bereits bei 65\%. Auch hier zeigt sich jedoch keine Steigerungsquote mehr im Vergleich zu den goer-Jahren (BZgA 2001). Bezüglich der AidsPrävention problematisch ist sicher nicht unbedingt das tatsächliche Infektionsrisiko der Cruppe der heterosexuellen, nicht-drogenabhängigen und nicht aus einem HPL (Hochprävalenzland mit HIV-Durchseuchung der Gesamtbevölkerung von > 1\% bei überwiegend heterosexueller Transmission z.B. in Schwarzafrika) stammenden Jugendlichen zwischen 15 und 19 Jahren: Die Quote dieser Altersgruppe an festgestellten Erstinfektionen beträgt insgesamt 1,8\% der männlichen HIV-Infizierten in 
Deutschland über die Jahre 1993-2003 hinweg. Davon wird wiederum im Mittel bei 12,6\% eine Ansteckung über heterosexuelle Kontakte vermutet (RKI 2003). Das Problem ergibt sich vielmehr durch die Weichenstellung für Verhaltensstile im späteren Sexualleben, eben weil Jugendliche diese Verhaltensweisen neu erlernen. Erwähnenswert ist hierbei, dass gerade die heterosexuellen Jugendlichen, die durch Promiskuität und ungeschützten Geschlechtsverkehr das stärkste Risikopotenzial aufweisen, die geringste Angst vor Aids haben, während die Nicht-Koitus-Erfahrenen als Gruppe mit dem geringsten Risiko diesbezüglich die stärksten Befürchtungen haben (vgl. Plies et al. 1999). Die hier zitierten Angaben sollten nicht darüber hinwegtäuschen, dass unter den Jugendlichen insgesamt eine recht große Bereitschaft zur Verhütung besteht. 71\% der Mädchen und 58\% der Jungen achten „,immer sehr genau“ darauf, dass die Kontrazeption beim Sexualverkehr gewährleistet ist (BZgA 2001). Über zwei Drittel der 14- bis 24-Jährigen haben in den letzten 12 Monaten „immer“ verhütet.

Von den Koituserfahrenen berichtet ein Drittel, beim Sex schon mal Angst vor der Infektion mit HIV gehabt zu haben. Knapp ein Drittel hat wiederum immer $(2,8 \%)$, oft $(7,8 \%)$ oder gelegentlich $(20,2 \%)$ Angst davor, dass beim Geschlechtsverkehr eine ungewollte Schwangerschaft eintritt. Dabei bestehen interessanterweise keine Unterschiede zwischen Mädchen und Jungen (Plies et al. 1999).

Im amerikanischen Schrifttum wird besonderes Augenmerk zurzeit darauf gelegt, welcher Art von sexueller Aktivität Jugendliche vor dem eigentlichen Geschlechtsverkehr nachgehen und inwieweit dabei eine Ansteckung mit sexuell übertragbaren Krankheiten (sexually transmitted diseases, STD) inklusive HIV droht. Dabei haftet den meisten Artikeln ein Unterton im Sinne einer Verurteilung jugendlicher Sexualität an, so etwa bei der Untersuchung von mit früher sexueller Aktivität sonst noch assoziierter verhaltensauffälliger Persönlichkeitsentwicklungen (Delinquenz, Drogen, niedriger sozialer Status, ethnische Stigmatisierung, vgl. Bailey et al. 1998; Burack 1999; Cohen et al. 2002; Cooksey et al. 2002; Gates u. Sonnenstein 200o; Kowaleski-Jones u. Mott 1998; Levy-Storms et al. 1998; O’Donnell et al. 2001; Li et al. 2001; Poulson et al. 1998; Ramirez-Valles et al. 1998; Sabo et al. 2001; Sen 2002; Stanton et al. 2001; Warren et al. 1998).

\subsection{Partnerschaftsverhalten und sexuelle Orientierung}

Im Kontext mit frühem sexuellem Risikoverhalten stehen Fragen zur Qualität und Beständigkeit der ersten sexuellen Partnerschaften bei Jugendlichen. Hierbei scheint insbesondere die Frage relevant, wie dauerhaft diese frühen Liebesbeziehungen angelegt sind bzw. wie tolerant die Gesellschaft mit diesen sexuellen Beziehungen umgeht, damit diese Bestand haben können. Nach den Umfragen der Bundeszentrale für gesundheitliche Aufklärung sind in „fester Beziehung“ immerhin etwa 35\% der Mädchen und 28\% der Jungen (BzgA 2001). Für das Eingehen erster sexueller Kontakte werden in Deutschland überwiegend das Bestehen einer „festen“ Beziehung oder doch zumindest einer intensiven Freundschaft angegeben. Spontane, relativ anonyme sexuelle Kontakte sind im heterosexuellen Bereich nach den Umfrageergebnissen der Bundeszentrale selten. Interessant ist die Einstellung zu homosexuellen Kontakten: Waren es 1980 noch etwa 10\% der Jungen, die gleichgeschlechtliche intensive Körpererfahrungen angaben, so liegt 2001 nach der Befragung der Bundeszentrale 
für gesundheitliche Aufklärung (BzgA 2001) die Prävalenz homosexuellen Verhaltens, ebenfalls definiert als „enger körperlicher Kontakt mit Gleichgeschlechtlichen“, nur noch bei 8\% der Mädchen und 5\% der Jungen.

Homosexuelle Erfahrungen sind im Jugendalter (Entwicklungshomosexualität) erfahrungsgemäß nicht im Sinne einer auf Dauer angelegten Fixierung auf die gleichgeschlechtliche Partnerwahl zu interpretieren. Oft wird die sexuelle Begierde gleichsam probehalber und aus Angst beziehungsweise Fremdheit (Hemmungshomosexualität) dem heterosexuellen Objekt gegenüber zunächst auf eine Person des gleichen Geschlechts gerichtet. Die „dauernde Inversion“ wird nach Freud normalerweise durch die natürliche Anziehung der entgegengesetzten Geschlechtscharaktere abgewehrt (Freud 1905). Dennoch sieht Freud homosexuelles Verhalten nicht automatisch als Perversion an, sondern als eine gesonderte Entwicklungslinie (Neigungshomosexualität) in der Wahl des sexuellen Objekts, in dem offenbar narzisstische Motive, im Sinne einer Spiegelung des eigenen Selbst, eine größere Rolle spielen, als die Ergänzung der Eigenschaften, wie dies für die heterosexuelle Objektwahl bestimmend zu sein scheint. Auch bei der homosexuellen Partnerschaft Jugendlicher scheint es von entscheidender Bedeutung zu sein, ob sexuelles Verhalten im Jugendalter auf genügend Toleranz aufseiten der Eltern, Erzieher und der Gesellschaft insgesamt trifft, um auf Dauer angelegt sein und damit die Risiken der Promiskuität vermeiden zu können.

\section{Fazit}

Pubertät und Adoleszenz sind also Phasen des Umbruchs, in denen die kindliche Psyche gleichsam eine erneute Entwicklungschance erhält. Dabei findet eine parallele Bewegung hin zur sexuellen wie zur psychosozialen Identitätsfindung statt. Der Diskurs der Sexualität (vgl. Foucault 1983) beschreibt das Ineinandergreifen von sexueller Selbstfindung und Identitätsentwicklung. Sexualität, sexuelle Objektliebe, die „Schule des Begehrens" formt die Persönlichkeit des Heranreifenden und schafft Identität durch Abgrenzung zu den Eltern unter Wahrung der Inzestschranke einerseits und durch die psychosexuelle Identitätsfindung bzgl. heterosexueller oder homosexueller Objektwahl andererseits. So ist die Adoleszenz eine Schule der seelischen Leidenschaften, ein „Entwicklungsroman“ des Individuums hin zur reifen Frau und zum erwachsenen Mann und markiert damit das endgültige Ende der Kindheit. Der einzige Ausgleich für diesen schweren Verlust des Heranreifenden ist die Möglichkeit zu jenen Sekunden der Lust, die Triebfeder unseres erwachsenen Lebens sind.

\section{Literatur}

Bailey SL, Camlin CS, Ennett ST (1998) Substance use and risky sexual behavior among homeless and runaway youth. I Adolesc Health 23, 378-88

Burack R (1999) Teenage sexual behaviour: attitudes towards and declared sexual activity. Br | Fam Plann 24, $145-8$

BzgA (2001) Bundeszentrale für gesundheitliche Aufklärung: Wiederholungsbefragung von 14-17-Jährigen und ihren Eltern. Ergebnisse der Repräsentativbefragung 2001

Clement U (1986) Sexualität im sozialen Wandel. Eine empirische Vergleichsstudie an Studenten 1966 und 1981. Enke Verlag Stuttgart

Cohen DA, Farley TA, Taylor SN, Martin DH, Schuster MA (2002) When and where do youth have sex? The potential role of adult supervision. Pediatrics 110, 6 oder online http://www.pediatrics.org/cgi/content/full/110/6/ e66 
Cooksey EC, Mott FL, Neubauer SA (2002) Friendships and early relationships: links to sexual initiation among American adolescents born to young mothers. Perspect Sex Reprod Health 34, 118-26

Euler S, Brosig B (2004) Sexuelle Aktivität in Pubertät und Adoleszenz. In: Schill WB, Weidner W, Bretzel G. (Hrsg.) Das Männerbuch. Urban und Fischer Verlag München, S. 96-107

Ewert 0 (1983) Entwicklungspsychologie des Jugendalters. Kohlhammer Verlag, Stuttgart

Foucault M (1983) Sexualität und Wahrheit. Suhrkamp Verlag Frankfurt

Fichte $H$ (1974) Versuch über die Pubertät. Hoffmann \& Campe Verlag Hamburg

Freud A (1958) Probleme der Pubertät. In: Freud A: Die Schriften der Anna Freud. Band IV (1956-1965). Fischer Verlag Frankfurt (1987), S.1739-1769

Freud S (1905) Die Umgestaltungen der Pubertät. In: Freud S: Gesammelte Werke. Band V (1904-1905). Fischer Verlag Frankfurt (1942), S. 109-159

Gates Gl, Sonnenstein FL (2000) Heterosexual genital sexual activity among adolescent males: 1988 and 1995. Fam Plann Perspect 32, 295-7

Gordon S, Gilgun I (1987) Adolescent sexuality. In: Van Hasselt V \& Hersen M (Eds.), Handbook of adolescent psychology. Pergamon Press Elmsford, NY

Kinsey AC, Pomeroy WB, Martin CE (1948) Sexual Behavior in the Human Male. Saunders Philadelphia. Deutsch: Kinsey Report. Das sexuelle Verhalten des Mannes. Fischer Verlag Frankfurt 1970

Kluge N (1998) Sexualverhalten Jugendlicher heute: Ergebnisse einer repräsentativen Jugend- und Elternstudie über Verhalten und Einstellungen zur Sexualität. Juventa Verlag Weinheim, München

Kowaleski-Jones I, Mott FL (1998) Sex, contraception and childbearing among high-risk youth: do different factors influence males and females? Fam Plann Perspect 30, 163-9

Lerner H (1987) Psychodynamic Models. In: Van Hasselt VB \& Hersen M. Handbook of Adolescent Psychology. Pergamon Press Oxford, S. 53-76

Levy-Storms L, Sucoff CA, Aneshensel CS (1998) Gender and ethnic differences in the timing of first sexual intercourse. Fam Plann Perspect 30, 121-7

Li X, Stanton B, Cottrell L, Burns I, Pack R, Kaljee L (2001) Patterns of initiation of sex and drug-related activities among urban low-income African-American adolescents. I Adolesc Health 28, 46-54

O'Donnell BL, O'Donnell CR, Stueve A (2001) Early sexual initiation and subsequent sex-related risks among urban minorità youth: the reach for health study. Fam Plann Perspect 33, 268-75

Plies K, Nickel B, Schmidt P (1999) Zwischen Lust und Frust. Jugendsexualität in den 9oer Jahren. Leske + Budrich Verlag Opladen

Poulson RL, Eppler MA, Satterwhite TN, Wuensch KL, Bass LA (1998) Alcohol consumption, strength of religous beliefs, and risky sexual behavior in college students. I Am Coll Health 46, 227-32

Ramirez-Valles J, Zimmermann MA, Newcomb MD (1998) Sexual risk behavior among youth: modelling the influence of prosocial activities and socioeconomic factors. I Health Soc Behav 39, 237-53

Rimbaud A (1907) Die Läusesucherinnen. Übersetzt von K Ammer. Insel Verlag Berlin. Zum Text siehe auch: http:// www.amadelio.de/weblog/2006/06/16/arthur-rimbaud-die-lausesucherinnen/, abgerufen am 02.08.2011

RKI (2003) Robert-Koch-Institut: Epidemiologisches Bulletin/Sonderausgabe B 11.08.2003

Sabo DF, Miller KE, Farrell MP, Melnick M), Barnes GM (2001) High school athletic participation, sexual behavior and adolescent pregnancy: a regional study. J Adolesc Health 25, 207-16

Schnack D, Neutzling R (1993) Die Prinzenrolle. Über die Männliche Sexualität. Rowohlt Verlag Reinbek

Schnack D, Neutzling R (1996) Kleine Helden in Not. Jungen auf der Suche nach Männlichkeit. Rowohlt Verlag Reinbek

Sen B (2002) Does alcohol-use increase the risk of sexual intercourse among adolescents? Evidence from the NLSY97. I Health Econ 21, 1085-93

Stanton B, Li X, Cottrell L, Kaljee L (2001) Early initiation of sex, drug related risk behaviors, and sensation-seeking among urban, low-income African-American adolescents. J Natl Med Assoc 93, 129-38

Trautner HM (1996) Die Bedeutung der Geschlechtskategorien im Jugendalter. In: Schumann-Hengsteler, R. \& Trautner, H.M.: Entwicklung im Jugendalter. Hogrefe Verlag Göttingen

Warren CW, Santelli JS, Everett SA, Kann L, Collins JL, Cassell C, Moprris L, Kolbe L) (1998) Sexual behavior among U.S. high school students, 1990-1995. Fam Plann Perspect 30, 170-2

Winnicott DW (1965) Maturational Processes and the Facilitating Environment. International Universities Press Madison 